\title{
REDUCIBLE FUNCTIONAL DIFFERENTIAL EQUATIONS
}

\author{
S.M. SHAH \\ Department of Mathematics \\ University of Kentucky \\ Lexington, Kentucky 40506 \\ and \\ JOSEPH WIENER \\ Department of Mathematics \\ Pan American University \\ Edinburg, Texas 78539 \\ (Received December 5, 1984)
}

ABSTRACT. This is the first part of a survey on analytic sclutions of functional differential equations (FDE). Some classes of FDE that can be reduced to ordinary differential equations are considered since they of ten provide an insight into the structure of analytic solutions to equations with more general argument deviations. Reducible FDE also find important applications in the study of stability of differential-difference equations and arise in a number of biological models.

KEY WORDS AID PHRASES. Functional Differential Equation, Argument Deviation, Involution.

1.980 MATHEMATICS SUBJECT CLASSIFICATION CODES. 34K05, 34K20, 34K9.9.

\section{INTRODUCTION.}

In [1-4] a method has been discovered for the study of a special class of functional differential equations -- differential equations with involutions. This basically algebraic approach was developed also in a number of other works and culminated in the monograph [5]. Though numerous papers continue to appear in this field [6-10], some aspects of the theory still require further investigation. In connection with the purposes of our article we mention only such topics as higher-order equations with rotation of the argument, equations in partial derivatives with involutions, influence of the method on the study of systems with deviations of more general nature, and solutions in spaces of generalized and entire functions. 


\section{DIFFERENTIAL EQUATIONS WITH INVOLUTIONS.}

In studying equations with a deviating argument, not only the general properties are of interest, but also the selection and analysis of the individual classes of such equations which admit of simple methods of investigation. In this section we consider a special type of functional differential equations that can be transformed into ordinary differential equations and thus provide an abundant source of relations with analytic solutions.

Silberstein [11] studied the equation

$$
x^{\prime}(t)=x\left(\frac{1}{t}\right), \quad 0<t<\infty .
$$

In [12] we proved that the solution is obtained very simply by a differentiation of (2.1). As a matter of fact,

$$
x^{\prime \prime}(t)=-\frac{1}{t^{2}} x^{\prime}\left(\frac{1}{t}\right)=-\frac{1}{t^{2}} x(t),
$$

whence,

$$
t^{2} x^{\prime \prime}(t)+x(t)=0 \text {. }
$$

Consequent $1 \mathrm{y}$,

$$
x(t)=\sqrt{t}\left[C_{1} \cos \left(\frac{\sqrt{3}}{2} \ln t\right)+C_{2} \sin \left(\frac{\sqrt{3}}{2} \ln t\right)\right] .
$$

Substituting $x(t)$ in (2.1), we obtain $C_{1}=\sqrt{3} C_{2}$, and finally,

$$
x(t)=C \sqrt{t} \cos \left(\frac{\sqrt{3}}{2} \ln t-\frac{\pi}{6}\right) \text {. }
$$

Obviously, the key to the solution is the fact that the function $f(t)=1 / t$ maps the interval $(0, \infty)$ one-to-one onto itself and that the relation

$$
f(f(t))=t,
$$

or, equivalently,

$$
f(t)=f^{-1}(t)
$$

is satisfied for each $t \&(0, \infty)$.

A function $f(t) \neq t$ that maps a set $G$ onto itself and satisfies on $G$ condition (2.3), is called an involution. In other words, an involution is a mapping which coincides with its own inverse. Let

$$
f_{1}(t)=f(t), f_{n+1}(t)=f\left(f_{n}(t)\right), \quad n=1,2, \ldots
$$

denote the iterations of a function $f: G \rightarrow G$. A function $f: G \rightarrow G$ is said to be an involution of order $m$ if there exists an integer $m \geq 2$ such that $f_{m}(t)=t$ for each 
$t \varepsilon G$, and $f_{n}(t) \not t$ for $n=1, \ldots, m-1$. It is easy to check that the following functions are involutions.

EXAMPLE 2.1. $f(t)=c-t$ on $R=(-\infty, \infty)$, where $c$ is an arbitrary real.

EXAMPLE 2.2.

$$
f(t)=\left\{\begin{array}{l}
-a t \text { for } t \geq 0, \\
-t / a \text { for } t \leq 0,
\end{array}\right.
$$

on $R$, where $a>0$ is arbitrary [5].

EXAMPLE 2.3.

$$
f(t)= \begin{cases}t^{-k} & \text { for } 0<t \leq 1 \\ -\frac{1}{k} & \text { for } t \geq 1\end{cases}
$$

on $(0, \infty)$, where $k$ is an arbitrary positive integer [5].

EXAMPLE 2.4. The function $f(z)=\varepsilon z$, where $\varepsilon=\exp (2 \pi i / m)$, is an involution of order $m$ on the complex plane.

EXAMPLE 2.5. The function [13]

$$
f(t)=\left\{\begin{array}{lll}
t, & t \varepsilon(-\infty, 0) U(m,+\infty), \\
t+1, & t \varepsilon(0,1) U(1,2) U \ldots U(m-2, m-1), \\
t-(m-1), & t \varepsilon(m-1, m)
\end{array}\right.
$$

is an involution of order $m$ on $G=(-\infty, 0) U(0,1) U \ldots U(m-1, m) U(m,+\infty)$.

DEFINITION 2.1. A real function $f(t) \neq t$ of a real variable $t$, defined on the whole axis and satisfying relation (2.3) for all $t$, is called a strong involution [1]

We denote the set of all such functions by $I$. The graph of each $f \varepsilon$ I is symmetric about the line $x=t$ in the $(t, x)$ plane. Conversely, if $\Gamma$ is the set of points of the $(t, x)$ plane, symmetric about the line $x=t$ and which contains for each $t$ a single point with abscissa $t$, then $\Gamma$ is a graph of a function from $I$. One of the methods for obtaining strong involutions is the following [14]. Assume that a real function $g(t, x)$ is defined on the set of all ordered pairs of real numbers and is such that if $g(t, x)=0$, then $g(x, t)=0$ (in particular, this is fulfilled if $g$ is symmetric, i.e., $g(t, x)=g(x, t))$. If to each $t$ there corresponds a single real $x=f(t)$ such that $g(t, x)=0$, then $f \varepsilon I$. For example,

$$
g(t, x)=t+x-c
$$

then

$$
f(t)=c-t
$$


If we take

$$
g(t, x)=t^{3}+x^{3}-c
$$

then

$$
f(t)=\sqrt[3]{c-t^{3}}
$$

Every continuous function $f \varepsilon I$ is strictly decreasing [15]. Hence,

$$
\lim _{t \rightarrow-\infty} f(t)=+\infty, \quad \lim _{t \rightarrow+\infty} f(t)=-\infty \text {. }
$$

THEOREM 2.1. A continuous strong involution $f(t)$ has a unique fixed point.

PROOF. The continuous function $\psi(t)=f(t)-t$ satisfies relations of the form (2.4) and, therefore, has a zero which is unique by virtue of its strict monotonicity. We also consider hyperbolic involutary mappings

$$
f(t)=\frac{\alpha t+\beta}{\gamma t-\alpha},\left(\alpha^{2}+\beta \gamma>0\right)
$$

which leave two points fixed. We introduce the following definition.

DEFINITION 2.2. A relation of the form

$$
F\left(t, x\left(f_{1}(t)\right), \ldots, x\left(f_{k}(t)\right), \ldots, x^{(n)}\left(f_{1}(t)\right), \ldots, x^{(n)}\left(f_{k}(t)\right)\right)=0,
$$

in which $f_{1}(t), \ldots, f_{k}(t)$ are involutions, is called a differential equation with involutions [1].

THEOREM $2.2([1])$. Let the equation

$$
x^{\prime}(t)=F(t, x(t), x(f(t)))
$$

satisfy the following hypotheses.

(i) The function $\mathrm{f}(\mathrm{t})$ is a continuously differentiable strong involution with a fixed point $t_{0}$.

(ii) The function $F$ is defined and is continuously differentiable in the whole space of its arguments.

(iii) The given equation is uniquely solvable with respect to $x(f(t))$ :

$$
x(f(t))=G\left(t, x(t), x^{\prime}(t)\right) .
$$

Then the solution of the ordinary differential equation

$x^{\prime \prime}(t)=\frac{\partial F}{\partial t}+\frac{\partial F}{\partial x(t)} x^{\prime}(t)+\frac{\partial F}{\partial x(f(t))} f^{\prime}(t) F(f(t), x(f(t)), x(t))$

(where $x(f(t)$ ) is given by expression (2.7)) with the initial conditions

$$
x\left(t_{0}\right)=x_{0}, \quad x^{\prime}\left(t_{0}\right)=F\left(t_{0}, x_{0}, x_{0}\right)
$$

is a solution of $\mathrm{Eq} .(2.6)$ with the initial condition

$$
x\left(t_{0}\right)=x_{0} \text {. }
$$


PROOF. Eq. (2.8) is obtained by differentiating (2.6). Indeed, we have

$x^{\prime \prime}(t)=\frac{\partial F}{\partial t}+\frac{\partial F}{\partial x(t)} x^{\prime}(t)+\frac{\partial F}{\partial x(f(t))} x^{\prime}(f(t)) f^{\prime}(t)$.

But from (2.6) and the relation $f(f(t))=t$ it follows that

$$
x^{\prime}(f(t))=F(f(t), x(f(t)), x(t)) \text {. }
$$

The second of the initial conditions (2.8') is a compatibility condition and is found from Eq. (2.6), with regard to (2.9) and $f\left(t_{0}\right)=t_{0}$. It is especially clear to see the role of involutions in equations which do not contain $t$ and $x(t)$ explicitly. In this case,

$$
x^{\prime}(f(t))=F(x(t))
$$

THEOREM 2.3 ([1]). Assume that in the equation

$$
x^{\prime}(t)=F(x(f(t)))
$$

the function $f(t)$ is a continuously differentiable strong involution with a fixed point $t_{0}$, and the function $F$ is defined, continuously differentiable, and strictly monotonic on $(-\infty, \infty)$.

Then the solution of the ordinary differential equation

$$
\begin{gathered}
x^{\prime \prime}(t)=F^{\prime}(x(f(t))) F(x(t)) f^{\prime}(t), \\
x(f(t))=F^{-1}\left(x^{\prime}(t)\right)
\end{gathered}
$$

with the initial conditions

$$
x\left(t_{0}\right)=x_{0}, \quad x^{\prime}\left(t_{0}\right)=F\left(x_{0}\right)
$$

is a solution of Eq. (2.10) with the initial condition $x\left(t_{0}\right)=x_{0}$.

COROLLARY. Theorems 2.2 and 2.3 remain valid if $f(t)$ is an involution of the form (2.5), while the equations are considered on one of the intervals $(-\infty, \alpha / \gamma)$ or $(\alpha / \gamma, \infty)$.

REMARK. Let $t_{0}$ be the fixed point of an involution $f(t)$. For $t>t_{0},(2.6)$ and (2.10) are retarded equations, whereas for $t<t_{0}$ they are of advanced type.

EXAMPLE 2.6. By differentiating the equation [1]

$$
x^{\prime}(t)=\frac{1}{x(a-t)}
$$

and taking into account that

$$
x^{\prime}(a-t)=\frac{1}{x(t)},
$$

we obtain the ordinary differential equation

$$
\frac{d^{2} x}{d t^{2}}=\frac{1}{x(t)}\left(\frac{d x}{d t}\right)^{2} \text {. }
$$


The fixed point of the involution $f(t)=a-t$ is $t_{0}=a / 2$. The initial condition for $(2.11)$ is

$$
x\left(\frac{a}{2}\right)=x_{0}
$$

the corresponding conditions for (2.12) are

$$
x\left(\frac{a}{2}\right)=x_{0}, \quad x^{\prime}\left(\frac{a}{2}\right)=\frac{1}{x_{0}} .
$$

Eq. (2.12) is integrable in quadratures:

$$
x(t)=x_{0} \exp \frac{t-\frac{a}{2}}{x_{0}^{2}}
$$

This is the solution of the original equation (2.11).

The topic of the paper [16] is the equation

$$
F\left(t, x(t), x^{\prime}(t), \ldots, x^{(n)}(t)\right)=x(f(t)),
$$

where $x$ is an unknown function.

THEOREM 2.4 ([16]). Let the following conditions be satisfied:

(1) The function $f$ maps the open set $G$ into $G$, $G$ being a subset of the set $R$ of real numbers.

(2) The function $f$ has iterations such that

$$
f_{1}(t)=f(t), \ldots, f_{k}(t)=f\left(f_{k-1}(t)\right), \ldots, f_{m}(t)=t
$$

for each $t \in G$, where $m$ is the smallest natural number for which the last expression holds.

(3) The function $f$ has derivatives up to, and inclusive of, the order $\mathrm{mn}-\mathrm{n}$ for each $t \varepsilon G, f^{\prime}(t) \neq 0$ for each $t \in G$.

(4) The function $F\left(t, u_{1}, u_{2}, \ldots, u_{n+1}\right)$ is $m n-n$ times differentiable of its arguments for each $t \varepsilon G$ and $u_{r} \varepsilon R(r=1, \ldots, n+1)$ and $\frac{\partial F}{\partial u_{n+1}} \neq 0$.

(5) The unknown function $x$ has derivatives up to, and inclusive of, the order mn on $G$.

In this case there exists an ordinary differential equation of order mn such that each solution of Eq. (2.13) is simultaneously a solution of this differential equation.

Let us consider the functional differential equation [17]

$F\left(f_{1}(t), x\left(f_{1}(t)\right), \ldots, x^{\left(k_{1}\right)}\left(f_{1}(t)\right), \ldots, x\left(f_{n}(t)\right), \ldots, x^{\left(k_{n}\right)}\left(f_{n}(t)\right)\right)=0,(2.14)$ where $x$ is an unknown function and where the following conditions are fulfilled: 
(1) The functions $f_{1}, \ldots, f_{n}$ form a finite group of order $n$ with respect to superposition of functions, $f_{1}(t)=t$, and map the open set $G$ into $G$, $G$ being the largest open set wherein all expressions appearing in this paper are defined.

(2) The functions $x$ and $f_{r}(r=1, \ldots, n)$ have derivatives up to the order $p$, where $p=\max \left(k_{1}, \ldots, k_{n}\right)$, so that $f_{r}^{\prime}(t) \neq 0$ for every $t \varepsilon G$ and $r=1, \ldots, n$.

(3) For the function $F$ at least one relation $\frac{\partial F}{\partial x^{(s)}\left(f_{r}\right)} \neq 0$ is valid for $s=0$, $\ldots, \mathrm{p} ; \mathrm{r}=2, \ldots, \mathrm{n}$ and every $\mathrm{t} \varepsilon \mathrm{G}$.

THEOREM 2.5 ([17]). If conditions (1)-(3) are satisfied, then every p-times differentiable solution of Eq. (2.14) is a component of the solution of a system of ordinary differential equations with argument $t$ only. This system is obtained from Fq. (2.14).

To investigate the equation $x^{\prime}(t)=f(x(t), x(-t))$, the author of [6] denotes $y(t)=x(-t)$ and obtains

$$
y^{\prime}(t)=-x^{\prime}(-t)=-f(x(-t), x(t))=-f(y, x) .
$$

Hence, the solutions of the original equation correspond to the solutions of the system of ordinary differential equations

$$
\frac{d x}{d t}=f(x, y), \quad \frac{d y}{d t}=-f(y, x)
$$

with the condition $x(0)=y(0)$. From the qualitative analysis of the solutions of the associated system he derives qualitative information about the solutions of the equation with transformed argument. The linear case is discussed in some detail. Several examples of more general equations are also considered.

Boundary-value problems for differential equations with reflection of the argument are studied in [10].

\section{LINEAR EQUATIONS}

In this section we study equations of the form

$$
L x(t) \equiv \sum_{k=0}^{n} a_{k}(t) x^{(k)}(t)=x(f(t))+\psi(t)
$$

with an involution $f(t)$.

THEOREM 3.1 ([1]). Suppose that the initial conditions

$$
x^{(k)}\left(t_{0}\right)=x_{k}, \quad k=0, \ldots, n-1
$$

are posed for Eq. (3.1) in which the coefficients $a_{k}(t)$, the function $\psi(t)$, and the strong (or hyperbolic (2.5)) involution $f(t)$ with fixed point $t_{0}$ belong to the class $c^{n}(-\infty, \infty)\left(\right.$ or $\left.C^{n}(\alpha / \gamma, \infty)\right)$. If $n>1$, then $f^{\prime}(t) \neq 0$. We introduce the operator 


$$
M=\frac{1}{f^{\prime}(t)} \frac{d}{d t}
$$

Then the solution of the linear ordinary differential equation

$$
\sum_{k=0}^{n} a_{k}(f(t)) M^{k} L x(t)-x(t)=\sum_{k=0}^{n} a_{k}(f(t)) M^{k} \psi(t)+\psi(f(t))
$$

with the initial conditions

$$
\begin{gathered}
x^{(k)}\left(t_{0}\right)=x_{k}, k=0, \ldots, n-1, \\
\left.M^{k} L x(t)\right|_{t=t_{0}}=x_{k}+\left.M^{k} \psi(t)\right|_{t=t_{0},}, k=0, \ldots, n-1
\end{gathered}
$$

is a solution of problem $(3,1)-(3,2)$.

PROOF. By successively differentiating (3.1) n times, we obtain

$$
\begin{gathered}
x(f(t))=L x(t)-\psi(t)=M^{0} L x(t)-M^{0} \psi(t), \\
x^{\prime}(f(t))=\frac{1}{f^{\prime}(t)} \frac{d}{d t} L x(t)-\frac{1}{f^{\prime}(t)} \frac{d}{d t} \psi(t)=M L x(t)-M \psi(t), \\
x^{\prime \prime}(f(t))=\frac{1}{f^{\prime}(t)} \frac{d}{d t} M L x(t)-\frac{1}{f^{\prime}(t)} \frac{d}{d t} M \psi(t)=M^{2} L x(t)-M^{2} \psi(t), \\
\cdot \cdot \cdot \cdot \cdot \cdot \cdot \cdot \cdot \cdot \cdot \cdot \cdot \cdot \cdot \cdot \cdot \cdot \cdot \cdot \\
x^{(n)}(f(t))=\frac{1}{f^{\prime}(t)} \frac{d}{d t} M^{n-1} L x(t)-\frac{1}{f^{\prime}(t)} \frac{d}{d t} M^{n-1} \psi(t)=M^{n} L x(t)-M^{n} \psi(t) .
\end{gathered}
$$

These relations are multiplied by $a_{0}(f(t)), a_{1}(f(t)), \ldots, a_{n}(f(t))$, respectively, and the results are added together:

$$
\sum_{k=0}^{n} a_{k}(f(t)) x^{(k)}(f(t))=\sum_{k=0}^{n} a_{k}(f(t)) M^{k} L x(t)-\sum_{k=0}^{n} a_{k}(f(t)) M^{k} \psi(t) .
$$

By virtue of $f(f(t))=t$, it follows from (3.1) that

$$
\sum_{k=0}^{n} a_{k}(f(t)) x^{(k)}(f(t))=x(t)+\psi(f(t)) \text {. }
$$

Thus, we obtain Eq. (3,4). In order that the solution of this equation satisfies problem (3.1)-(3.2), we need to pose the following initial conditions for $(3.4)$ : the values of the function $x(t)$ and of its $n-1$ derivatives at the point $t_{0}$ should equal $x_{k}, k=0, \ldots, n-1$, from $(3.2)$, while the values $x^{(n)}\left(t_{0}\right), \ldots, x^{(2 n-1)}\left(t_{0}\right)$ are determined from the relations

$$
M^{k} L x(t)=x^{(k)}(f(t))+M^{k} \psi(t), k=0, \ldots, n-1
$$

by substituting the values $t_{0}$ and $x_{k}$ for $t$ and $x^{(k)}(t)$. 
THEOREM 3.2 ([1]). The equation

$$
x^{(n)}(t)=x\left(\frac{1}{t}\right)
$$

is integrable in quadratures and has a fundamental system of solutions of the form

$$
t^{a}(\ln t)^{j} \sin (b \ln t), t^{a}(\ln t)^{j} \cos (b \ln t),
$$

$a$ and $b$ are real and $j$ is a nonnegative integer.

PROOF. By an n-fold differentiation Eq. (3.6) is reduced to the Euler equation

$$
\sum_{k=n+1}^{2 n} b_{k}^{(n)} t^{k} x^{(k)}(t)=x(t)
$$

For $\mathrm{n}=1$ this follows from (2.2). Let us assume that the assertion is true for $\mathrm{n}$ and prove its validity for $n+1$. In accordance with formula (3.3), we introduce for Eq. (3.6) the operator

$$
M=-t^{2} \frac{d}{d t}
$$

On the basis of (3.4) and (3.8) we have

$$
\begin{aligned}
M^{n} x^{(n)}(t) & =\sum_{k=n+1}^{2 n} b_{k}^{(n)} t k_{x}(k)(t), \\
M^{n} x^{(n+1)}(t) & =\sum_{k=n+1}^{2 n} b_{k}^{(n)} t k_{x}(k+1)(t) .
\end{aligned}
$$

Then

$$
\begin{aligned}
& M^{n+1} x^{(n+1)}(t)=-t^{2} \frac{d}{d t} \sum_{k=n+1}^{2 n} b_{k}^{(n)} t^{k} x^{(k+1)}(t)= \\
& =-\sum_{k=n+1}^{2 n} k b_{k}^{(n)} t^{k+1} x^{(k+1)}(t)-\sum_{k=n+1}^{2 n} b_{k}^{(n)} t^{k+2} x^{(k+2)}(t) .
\end{aligned}
$$

Consequently, the equation

$$
x^{(n+1)}(t)=x\left(\frac{1}{t}\right)
$$

is reduced by an $(n+1)$-fold differentiation to the Euler equation

$$
M^{n+1} x^{(n+1)}(t)=x(t)
$$

At the same time we established the recurrence relation

$$
\begin{gathered}
b_{k}^{(n+1)}=-(k-1) b_{k-1}^{(n)}-b_{k-2}^{(n)}, n+2 \leq k \leq 2 n+2, \\
b_{n}^{(n)}=0, \quad b_{2 n+1}^{(n)}=0,
\end{gathered}
$$

connecting the coefficients of the Euler equations

$$
\sum_{k=n+1}^{2 n} b_{k}^{(n)} t^{k} x^{(k)}(t)=x(t) \text { and } \sum_{k=n+2}^{2 n+2} b_{k}^{(n+1)} t^{k} x^{(k)}(t)=x(t) \text {, }
$$


which correspond to the equations

$$
x^{(n)}(t)=x\left(\frac{1}{t}\right) \text { and } x^{(n+1)}(t)=x\left(\frac{1}{t}\right) .
$$

It is well known [18] that the Euler equation has a fundamental system of solutions of the form (3.7), where $a+b i$ is a root of the characteristic equation and $j$ is $a$ nonnegative integer smaller than its multiplicity. The theorem is proved.

EXAMPLE 3.1. The investigation of the nonhomogeneous equation [1]

$$
\begin{aligned}
x^{\prime}(t)= & x\left(\frac{1}{t}\right)+\psi(t), 0<t<\infty, \psi(t) \varepsilon c^{1}(0, \infty) \\
& x(1)=x_{0}
\end{aligned}
$$

reduces to the problem

$$
\begin{aligned}
& t^{2} x^{\prime \prime}(t)+x(t)=t^{2} \psi^{\prime}(t)-\psi\left(\frac{1}{t}\right), \\
& x(1)=x_{0}, \quad x^{\prime}(1)=x_{0}+\psi(1) .
\end{aligned}
$$

The solution is

$$
\begin{aligned}
x(t)= & x_{0} \sqrt{t} \cos \left(\frac{\sqrt{3}}{2} \ln t\right)+\frac{2}{\sqrt{3}}\left(\frac{x_{0}}{2}+\psi(1)\right) \sqrt{t} \sin \left(\frac{\sqrt{3}}{2} \ln t\right)+ \\
& \frac{2 \sqrt{t}}{\sqrt{3}} \int_{1}^{t} u^{-3 / 2} \sin \left(\frac{\sqrt{3}}{2} \ln \frac{t}{u}\right)\left[u^{2} \psi^{\prime}(u)-\psi\left(\frac{1}{u}\right)\right] d u .
\end{aligned}
$$

THEOREM 3.3 ([1]). The solution of the equation

$$
\begin{gathered}
x^{\prime}(t)=\alpha t^{\beta} x\left(\frac{1}{t}\right), \quad 0<t<\infty, \\
x(1)=x_{0}
\end{gathered}
$$

is representable in closed form.

PROOF. After differentiation Eq. (3.9) is reduced to the form

$$
t^{2} x^{\prime \prime}(t)-\beta t x^{\prime}(t)+\alpha^{2} x(t)=0
$$

with the initial conditions

$$
x(1)=x_{0}, \quad x^{\prime}(1)=\alpha x_{0} .
$$

Putting $t=\exp u$, we obtain

$$
x^{\prime \prime}(u)-(1+\beta) x^{\prime}(u)+\alpha^{2} x(u)=0,
$$

The roots of the characteristic equation

are

$$
\lambda^{2}-(1+\beta) \lambda+\alpha^{2}=0
$$

$$
\lambda_{1,2}=\frac{1+\beta}{2} \pm \sqrt{\frac{(1+\beta)^{2}}{4}-\alpha^{2}} \text {. }
$$

Consider various cases:

(1) $\frac{(1+\beta)^{2}}{4}-\alpha^{2} \equiv \Delta^{2}>0$, 


$$
x(t)=\frac{x_{0}}{\lambda_{1}-\lambda_{2}}\left[\left(\alpha-\lambda_{2}\right) t^{\lambda_{1}}+\left(\lambda_{1}-\alpha\right) t^{\lambda_{2}}\right] .
$$

(2) $\frac{(1+B)^{2}}{4}-\alpha^{2}=0$,

$$
x(t)=x_{0} t^{(1+\beta) / 2}\left[1+\left(\alpha-\frac{1+\beta}{2}\right) 1 n t\right] .
$$

(3) $\frac{(1+B)^{2}}{4}-\alpha^{2} \equiv-\Delta^{2}<0$,

$$
x(t)=x_{0} t^{(1+\beta) / 2}\left[\cos (\Delta \ln t)+\frac{\alpha-\frac{1+\beta}{2}}{\Delta} \sin (\Delta \ln t)\right] .
$$

For $\alpha=1, \beta=0$ the latter formula yields the solution of the Silberstein equation (2.1).

EXAMPLE 3.2. The equation

$$
a t^{r} x(t)+b t^{r+1} x^{\prime}(t)=c t^{s+1} x\left(\frac{1}{t}\right)+d t^{s} x^{\prime}\left(\frac{1}{t}\right),(t>0)
$$

where $a, b, c, d, r, s$ are real constants and $x$ is an unknown function, was explored in $[17]$.

Let $b^{2}-d^{2} \neq 0$. By putting $x\left(\frac{1}{t}\right)=y(t)$, Eq. $(3,10)$ becomes

$$
d t^{r} x(t)+b t^{r+1} x^{\prime}(t)=c t^{s+1} y(t)-d t^{s+2} y^{\prime}(t) \text {. }
$$

If $\frac{1}{t}$ is substituted for $t$ in (3.10), we get

$$
a t^{-r} y(t)-b t^{-r+1} y^{\prime}(t)=c t^{-s-1} x(t)+d t^{-s} x^{\prime}(t) \text {. }
$$

From (3.11) and (3.12) Euler's equation is obtained:

$$
t^{2} x^{\prime \prime}(t)+(r-s) t x^{\prime}(t)+\left(B s-B r+A^{2}-B^{2}+B\right) x(t)=0,
$$

where $A=(b c-a d) /\left(b^{2}-d^{2}\right), B=(c d-a b) /\left(b^{2}-d^{2}\right)$.

If $b=d$ and $a=c, E q .(3.10)$ is equivalent to the system of equations

$$
a x(t)+b t x^{\prime}(t)=u(t), \quad u\left(\frac{1}{t}\right)=t^{r-s-1} u(t) .
$$

If $b=d$ and $a \neq c,(3.10)$ reduces to the functional equation

$$
x\left(\frac{1}{t}\right)=t^{r-s-1} x(t) .
$$

In the case of $b=-d$ and $a=-c$ Eq. (3.10) reduces to the system

$$
a x(t)+b t x^{\prime}(t)=u(t), \quad u\left(\frac{1}{t}\right)=-t^{r-s-1} u(t) .
$$

In the case of $b=-d$ and $a \neq-c(3.10)$ reduces to the functional equation

$$
x\left(\frac{1}{t}\right)=-t^{r-s-1} x(t) .
$$

The equation $x^{\prime}(t)=x(f(t))$ with an involution $f(t)$ has been studied in [19]. 
Consider the equation [13] with respect to the unknown function $x(t)$ :

$$
x^{\prime}(t)=a(t) x(f(t))+b(t),
$$

(1) The function $f$ maps an open set $G$ onto $G$.

(2) The function $f$ can be iterated in the following way:

$$
f_{1}(t)=f(t), \ldots, f_{k}(t)=f\left(f_{k-1}(t)\right), \ldots, f_{m}(t)=t,(t \in G)
$$

where $m$ is the least natural number for which the last relation holds.

(3) The functions $a(t), b(t)$ and $f(t)$ are $m-1$ times differentiable on $G$, and $x(t)$ is $m$ times differentiable on the same set.

THEOREM 3.4 ( [13]). Eq. (3.13), for which conditions (1) - (3) hold, can be reduced to a linear differential equation of order $\mathrm{m}$.

EXAMPLE 3.3. Consider the equation [16]

$$
x^{\prime}(t)=x(f(t)), f(t)=(1-t)^{-1},
$$

and $G=(-\infty, 0) \mathrm{U}(0,1) \mathrm{U}(1,+\infty)$. For $\mathrm{f}$ we have $\mathrm{f}_{3}(t)=t$ on $G$. In this case $(3.14)$ is reducible to the equation

$$
t^{2}(1-t)^{2} x^{\prime \prime \prime}(t)-2 t^{2}(1-t) x^{\prime \prime}(t)-x(t)=0 .
$$

THEOREM 3.5 ([1]). In the system

$$
x^{\prime}(t)=A x(t)+B x(c-t), x(c / 2)=x_{0}
$$

let $\mathrm{A}$ and $\mathrm{B}$ be constant commutative $\mathrm{r} \times \mathrm{r}$ matrices, $\mathrm{x}$ be an $\mathrm{r}$-dimensional vector, and $B$ be nonsingular.

Then the solution of the system

$$
\begin{gathered}
x^{\prime \prime}(t)=\left(A^{2}-B^{2}\right) x(t), \\
x(c / 2)=x_{0}, \quad x^{\prime}(c / 2)=(A+B) x_{0}
\end{gathered}
$$

is the solution of problem (3.15).

In [7] it has been proved that the equation

$$
t^{2} x^{\prime \prime}(t)-\sqrt{12} \times\left(\frac{1}{t}\right)=0,0<t<\infty
$$

has the general solution

$$
x(t)=c_{1}\left(\sqrt{3} t^{2}+t^{-2}\right)+c_{2}\left[\sin (\sqrt{3} \ln t)-\frac{\sqrt{3} \cos (\sqrt{3} \ln t)}{3+\sqrt{12}}\right],
$$

while the equation

$$
t^{2} x^{\prime \prime}(t)+\sqrt{12} \times\left(\frac{1}{t}\right)=0
$$

has the general solution

$$
x(t)=c_{3}\left(t^{2}-\sqrt{3} t^{-2}\right)+c_{4}\left[\sin (\sqrt{3} \ln t)+\frac{3+\sqrt{12}}{\sqrt{3}} \cos (\sqrt{3} \ln t)\right] .
$$


It fullows from here that, by appropriate choice of $c_{1}, c_{2}, c_{3}$, and $c_{4}$, we can obtain both oscillating and nonoscillating solutions of the above equations. On the other hand, it is known that, for ordinary second-order equations, all solutions are either simultaneously oscillating or simultaneously nonoscillating. It has been also proved in [7] that the system

$$
\begin{aligned}
& x^{\prime}(t)=A(t) x(t)+f\left(t, x\left(\frac{1}{t}\right)\right), \quad 1 \leq t<\infty, \\
& \left\|f\left(t, x\left(\frac{1}{t}\right)\right)\right\| \leq \delta\left\|x\left(\frac{1}{t}\right)\right\|^{q},
\end{aligned}
$$

where $\delta>0$ and $q \geq 1$ are constants, is stable with respect to the first approximation.

For the equation

$$
\sum_{k=0}^{n} a_{k} t^{k}(k)(t)=x\left(\frac{1}{t}\right), \quad 0<t<\infty
$$

we prove the following result.

THEOREM 3.6. Eq.(3.16) is reducible by the substitution $t=e^{s}$ to a linear ordinary differential equation with constant coefficients and has a fundamental system of solutions of the form (3.7).

PROOF. Put $t=e^{S}$ and $x\left(e^{s}\right)=y(s)$, then $t x^{\prime}(t)=y^{\prime}(s)$. Assume that

$$
t^{k} x^{(k)}(t)=L y(s) \text {, }
$$

where $L$ is a linear differential operator with constant coefficients. From the relation

$$
t^{k+1} x^{(k+1)}(t)=t \frac{d}{d t}\left[t^{k} x^{(k)}(t)\right]-k t^{k} x^{(k)}(t)
$$

we obtain

$$
t^{k+1} x^{(k+1)}(t)=L\left[y^{\prime}(s)-k y(s)\right]
$$

which proves the assertion.

The functional differential equation

$$
\mathrm{Q}^{\prime}(\mathrm{t})=\mathrm{AQ}(\mathrm{t})+\mathrm{B} \mathrm{Q}^{\mathrm{T}}(\tau-\mathrm{t}),-\infty<\mathrm{t}<\infty,
$$

where $A, B$ are $n \times n$ constant matrices, $\tau \geq 0, Q(t)$ is a differentiable $n \times n$ matrix and $Q^{T}(t)$ is its transpose, has been studied in [20]. Existence, uniqueness and an algebraic representation of its solutions are given. This equation, of considerable interest in its own right, arises naturally in the construction of Liapunov functionals for retarded differential equations of the form $x^{\prime}(t)=C x(t)+D x(t-\tau)$, where $C, D$ are constant $n \times n$ matrices. The role played by the matrix $Q(t)$ is analogous to the one played by a positive definite matrix in the construction of Liapunov functions 
for ordinary differential equations. It is shown that, unlike the infinite dimensionality of the vector space of solutions of functional differential equations, the linear vector space of solutions to (3.17) is of dimension $n^{2}$. Moreover, the authors give a complete algebraic characterization of these $n^{2}$ linearly independent solutions which parallels the one for ordinary differential equations, indicate computationally simple methods for obtaining the solutions, and allude to the variation of constants formula for the nonhomogeneous problem.

The initial condition for $(3.17)$ is

$$
\mathrm{Q}\left(\frac{\tau}{2}\right)=\mathrm{K},
$$

where $K$ is an arbitrary $n \times n$ matrix. Eq. (3.17) is intimately related to the system

$$
\begin{aligned}
& Q^{\prime}(t)=A Q(t)+B R(t), \\
& R^{\prime}(t)=-Q(t) B^{T}-R(t) A^{T},
\end{aligned}
$$

with the initial conditions

$$
Q\left(\frac{\tau}{2}\right)=K, \quad R\left(\frac{\tau}{2}\right)=K^{T} \text {. }
$$

For any two $n \times n$ matrices $P, S$, let the $n^{2} \times n^{2}$ matrix $P \otimes S$ denote the Kronecker (or direct) product [21] and introduce the notation for the $\mathrm{n} \times \mathrm{n}$ matrix

$$
s=\left(s_{i j}\right)=\left(\frac{s_{1} *}{s_{n} *}\right)=\left[\begin{array}{c}
s_{1 *} \\
\dot{1} \\
\dot{s}_{n^{*}}
\end{array}\right]
$$

where $s_{i^{*}}$ and $s_{*_{j}}$ are, respectively, the $i$ th row and the $j$ th column of $s$; further, let there correspond to the $n \times n$ matrix $s$ the $n^{2}$-vector $s=\left(s_{1}, \ldots, s_{n}\right)^{T}$. With this notation Eqs. (3.19) and (3.20) can be rewritten as

and

$$
\left[\begin{array}{l}
q(t) \\
r(t)
\end{array}\right]^{\prime}=\left[\begin{array}{cc}
A \otimes I & B \otimes I \\
-I \otimes B & -I \otimes_{A}
\end{array}\right]\left[\begin{array}{l}
q(t) \\
r(t)
\end{array}\right]
$$

$$
q\left(\frac{\tau}{2}\right)=\left[k_{1 *}, \ldots, k_{n *}\right]^{T}, \quad r\left(\frac{\tau}{2}\right)=\left[k_{* 1}^{T}, \ldots, k_{*_{n}}^{T}\right]^{T},
$$

which, with the obvious correspondence and for simplicity of notation, are denoted as

$$
p^{\prime}(t)=C p(t), \quad p(\tau / 2)=p_{\tau / 2} \text {. }
$$

Here $p(t)$ is an $2 n^{2}$-vector and $c$ is a $2 n^{2} \times 2 n^{2}$ constant matrix. (3.21) is used in proving the following result:

THEOREM 3.7 ([20]). Eq. (3.17) with the initial condition (3.18) has a unique solution $Q(t)$ for $-\infty<t<\infty$.

Examination of the proof makes it clear that knowledge of the solution to (3.21) 
immediately yields the solution of (3.17)-(3.18). But (3.21) is a standard initialvalue problem in ordinary differential equations; the structure of the solutions of such problems is well known. Furthermore, since the $2 n^{2} \times 2 n^{2}$ matrix $C$ has a very special structure, it is possible to recover the structure of the solutions of Eq. (3.17). Let $\lambda_{1}, \ldots, \lambda_{p}, p=2 n^{2}$, be the distinct eigenvalues of the matrix $c$, that is, solutions of the determinantal equation

$$
\operatorname{det}[\lambda I-C]=0 \text {, }
$$

each $\lambda_{j}, j=1, \ldots, p$, with algebraic multiplicity $m_{j}$ and geometric multiplicities $n_{j}^{r}, \sum_{r=1}^{s} n_{j}^{r}=m_{j}, \sum_{j} m_{j}=2 n^{2}$. Then $2 n^{2}$ linearly independent solutions of (3.21) ar $\epsilon$ given by

$$
\psi_{j, r} q(t)=\exp \left(\lambda_{j}\left(t-\frac{\tau}{2}\right)\right) \sum_{i=1}^{q} \frac{\left(t-\frac{\tau}{2}\right)^{q-i}}{(q-i) !} e_{j, r},
$$

where $q=1, \ldots, n_{j}^{r}$, and the $2 n^{2}$ linearly independent eigenvectors and generalized eigenvectors are given by

$$
\left[\lambda_{j} I-C\right] e_{j, r} i=-e_{j, r}^{i-1}, e_{j, s}^{0}=0 .
$$

A change of notation, and a return from the vector to the matrix form, shows that $2 n^{2}$ linearly independent solutions of (3.19) are given by

$$
\left[\begin{array}{l}
\Psi_{j, r},(t) \\
Y_{j, r}^{q}(t)
\end{array}\right]=\exp \left(\lambda_{j}\left(t-\frac{\tau}{2}\right)\right) \sum_{i=1}^{q} \frac{\left(t-\frac{\tau}{2}\right)^{q-i}}{(q-i) !}\left[\begin{array}{r}
L_{j, r}^{i} \\
M_{j, r}^{i}
\end{array}\right]
$$

where the generalized eigenmatrix pair $\left(L_{j, r}^{i}, M_{j, r}^{i}\right)$ associated with the eigenvalue $\lambda_{j}$ satisfies the equations

$$
\begin{aligned}
& \left(\lambda_{j} I-A\right) L_{j, r}^{i}-B M_{j, r}^{i}=-L_{j, r}^{i-1}, \\
& L_{j, r} B^{T}+M_{j, r}^{i}\left(\lambda_{j} I+A^{T}\right)=-M_{j, r}^{i-1} .
\end{aligned}
$$

The structure of these equations is a most particular one; indeed, if they are multiplied by -1 , transposed, and written in reverse order, they yield

$$
\begin{aligned}
& \left(-\lambda_{j} I-A\right) M_{j, r} i^{T}-B L_{j, r} i^{T}=M_{j, r} r^{T} \\
& M_{j, r}{ }^{T} B_{j, r}^{i^{T}}+L_{j, r} i^{T}\left(-\lambda, I+A^{T}\right)=L_{j, r} r^{T},
\end{aligned}
$$

$L_{j, r} 0^{T}=M_{j, r} 0^{T}=0$. But this result demonstrates that if $\lambda_{j}$ is a solution of (3.22), $-\lambda_{j}$ will also be a solution; moreover, $\lambda_{j}$ and $-\lambda_{j}$ have the same geometric multiplici- 
ties and the same algebraic multiplicity. Hence, the distinct eigenvalues always appear in pairs $\left(\lambda_{j},-\lambda_{j}\right)$, and if the generalized eigenmatrix pairs corresponding to $\lambda_{j}$ are $\left(L_{j}, r, M_{j}, r\right)$, the generalized eigenmatrix pairs corresponding to $-\lambda_{j}$ will be $\left((-1)^{i+1} M_{j, r} i^{T},(-1)^{i+1} L_{j, r} i^{T}\right)$. These remarks imply that if the solution (3.23) corresponding to $\lambda_{j}$ is added to the solution (3.23) corresponding to $-\lambda_{j}$ multiplied by $(-1)^{q+i}$, the $n^{2}$ linearly independent solutions of (3.19) given by

$$
\begin{array}{r}
{\left[\begin{array}{l}
z_{j, r}(t) \\
w_{j, r}{ }^{q}(t)
\end{array}\right]=\exp \left(\lambda_{j}\left(t-\frac{\tau}{2}\right)\right) \sum_{i=1}^{q} \frac{\left(t-\frac{\tau}{2}\right)^{q-i}}{(q-i) !}\left[\begin{array}{r}
L_{j, r} \\
M_{j, r}^{i}
\end{array}\right]+} \\
\exp \left(-\lambda_{j}\left(t-\frac{\tau}{2}\right)\right) \sum_{i=1}^{q} \frac{\left(t-\frac{\tau}{2}\right)^{q-i}}{(q-i) !}(-1)^{q+i}\left[\begin{array}{c}
M_{j, r} i^{T} \\
i_{j, r}^{T}
\end{array}\right]
\end{array}
$$

satisfy the condition

$$
z_{j, r}\left(\frac{\tau}{2}\right)=w_{j, r} q^{T}\left(\frac{\tau}{2}\right)
$$

But this is precisely condition (3.20); it therefore follows that the expressions

$$
\begin{aligned}
& z_{j, r} q(t)=\sum_{i=1}^{q} \frac{\left(t-\frac{\tau}{2}\right)^{q-i}}{(q-i) !}\left[\exp \left(\lambda_{j}\left(t-\frac{\tau}{2}\right)\right) L_{j, r}{ }^{i}+\right. \\
& \left.(-1)^{q+i} \exp \left(-\lambda_{j}\left(t-\frac{\tau}{2}\right)\right) M_{j, r} i^{T}\right]
\end{aligned}
$$

are $\mathrm{n}^{2}$ 1inearly independent solutions of (3.17).

THEOREM 3.8 ([20]). Eq. (3.17) has $\mathrm{n}^{2}$ linearly independent solutions given by Eq. (3.25), where the generalized eigenmatrix pairs $\left(L_{j, r}, M_{j, r}\right)$ satisfy Eq. (3.24) for one of the elements of the pair $\left(\lambda_{j},-\lambda_{j}\right)$, each of which is a solution of Eq. $(3.22)$.

Eq. (3.17) has been used in [22] for the construction of Liapunov functionals and also encountered in a somewhat different form in [23].

Some problems of mathematical physics lead to the study of initial and boundary value problems for equations in partial derivatives with deviating arguments. Since research in this direction is developed poorly, the investigation of equations with involutions is of certain interest. They can be reduced to equations without argument deviations and, on the other hand, their study discovers essential differences 
that may appear between the behavior of solutions to functional differential equations and the corresponding equations without argument deviations.

The solution of the mixed problem with homogeneous boundary conditions and initial values at the fixed point $t_{0}$ of the involution $f(t)$ for the equations

$$
u_{t}(t, x)=a u_{x x}(t, x)+b u_{x x}(f(t), x) \text {, }
$$

and

$$
u_{t t}(t, x)=a^{2} u_{x x}(t, x)+b^{2} u_{x x}(f(t), x)
$$

can be found by the method of separation of the variables. Thus, for (3.26) the functions $T_{n}(t)$ in the expansion

$$
u(t, x)=\sum_{n=1}^{\infty} T_{n}(t) x_{n}(x)
$$

are determined from the relation

$$
T_{n}^{\prime}(t)=-\lambda_{n} a T_{n}(t)-\lambda_{n} b T_{n}(f(t)), T_{n}\left(t_{0}\right)=C_{n} .
$$

Its investigation is carried out by means of Theorem 3.1 , according to which the solution of the equation

$$
T_{n}^{\prime \prime}(t)=-\lambda_{n} a\left(1+f^{\prime}(t)\right) T_{n}^{\prime}(t)-\lambda_{n}^{2}\left(a^{2}-b^{2}\right) f^{\prime}(t) T_{n}(t)
$$

with the initial conditions

$$
T_{n}\left(t_{0}\right)=C_{n}, \quad T_{n}^{\prime}\left(t_{0}\right)=-\lambda_{n}(a+b) C_{n}
$$

satisfies Eq. (3.29). The following theorems illustrate striking dissimilarities between equations of the form (3.26) and (3.27) and the corresponding equations without argument deviations.

THEOREM 3.9. The solution of the problem

$$
\begin{aligned}
& u_{t}(t, x)=a u_{x x}(t, x)+b u_{x x}(c-t, x), \\
& u(t, 0)=u(t, l)=0, u(c / 2, x)=\psi(x)
\end{aligned}
$$

is unbounded as $t \rightarrow \pm \infty$ if $a=b \neq 0$. If $|b|<|a|, b \neq 0$, expansion $(3,28)$ diverges for all $t \neq c / 2$.

PROOF. By separating the variables, we obtain

$$
T_{n}^{\prime}(t)=-\frac{\pi^{2} n^{2}}{l^{2}}\left(a T_{n}(t)+b T_{n}(c-t)\right), T_{n}(c / 2)=C_{n} \text {. }
$$

The initial conditions for equations (3.31) and (3.32) are posed at the fixed point of the involution $f(t)=c-t$. In this case, Eq. (3.30) takes the form

$$
\begin{aligned}
T_{n}^{\prime \prime}(t) & =-\frac{\pi^{4} n^{4}}{\ell^{4}}\left(b^{2}-a^{2}\right) T_{n}(t), \\
T_{n}(c / 2) & =C_{n}, T_{n}^{\prime}(c / 2)=-\frac{\pi^{2} n^{2}}{\ell^{2}}(a+b) C_{n} .
\end{aligned}
$$


The completion of the proof is a result of simple computations. Depending on the relations between the coefficients $a$ and $b$, the following possibilities may occur:

(1) $T_{n}(t)=c_{n}\left(\cos \frac{\pi^{2} n^{2} \sqrt{b^{2}-a^{2}\left(t-\frac{c}{2}\right)}}{l^{2}}-\right.$

$$
\left.\frac{a+b}{\sqrt{b^{2}-a^{2}}} \sin \frac{\pi^{2} n^{2} \sqrt{b^{2}-a^{2}}\left(t-\frac{c}{?}\right)}{l^{2}}\right),(|a|<|b|) \text {; }
$$

(2) $T_{n}(t)=c_{n}\left(1-\frac{\pi^{2} n^{2}}{\ell^{2}}(a+b)\left(t-\frac{c}{2}\right)\right),(|a|=|b|)$;

(3) $T_{n}(t)=\frac{1}{2} C_{n}\left[\left(1-\frac{\sqrt{a^{2}-b^{2}}}{a-b}\right) \exp \left(\frac{\pi^{2} n^{2}}{l^{2}} \sqrt{a^{2}-b^{2}}\left(t-\frac{c}{2}\right)\right)+\right.$

$$
\left.\left(1+\frac{\sqrt{a^{2}-b^{2}}}{a-b}\right) \exp \left(-\frac{\pi^{2} n^{2}}{\ell^{2}} \sqrt{a^{2}-b^{2}}\left(t-\frac{c}{2}\right)\right)\right],(|b|<|a|) .
$$

IHEOREM 3.10. The solution of the equation

$$
u_{t t}(t, x)=a^{2} u_{x x}(t, x)+b^{2} u_{x x}(-t, x)
$$

satisfying the boundary and initial conditions

$$
u(t, 0)=u(t, \ell)=0, u(0, x)=\phi(x), u_{t}(0, x)=\psi(x),
$$

is unbounded as $t \rightarrow \pm \infty$ if $a^{2}=b^{2}$. In the case $a^{2}<b^{2}$ expansion (3.28) diverges for all $t \neq 0$.

PROOF. Separation of the variables gives for the functions $T_{n}(t)$ the relation

$$
\begin{gathered}
T_{n}^{\prime \prime}(t)=-\frac{\pi^{2} a^{2} n^{2}}{l^{2}} T_{n}(t)-\frac{\pi^{2} b^{2} n^{2}}{\ell^{2}} T_{n}(-t), \\
T_{n}(0)=A_{n}, \quad T_{n}^{\prime}(0)=B_{n},
\end{gathered}
$$

by successive differentiation of which we obtain

$$
\begin{aligned}
& T_{n}^{(3)}(t)=-\frac{\pi^{2} a^{2} n^{2}}{\ell^{2}} T_{n}^{\prime}(t)+\frac{\pi^{2} b^{2} n^{2}}{\ell^{2}} T_{n}^{\prime}(-t), \\
& T_{n}^{(4)}(t)=-\frac{\pi^{2} a^{2} n^{2}}{\ell^{2}} T_{n}^{\prime \prime}(t)-\frac{\pi^{2} b^{2} n^{2}}{\ell^{2}} T_{n}^{\prime \prime}(-t) .
\end{aligned}
$$

From Eq. (3.33) we find

$$
T_{n}^{\prime \prime}(-t)=-\frac{\pi^{2} a^{2} n^{2}}{l^{2}} T_{n}(-t)-\frac{\pi^{2} b^{2} n^{2}}{l^{2}} T_{n}(t)
$$

and also

$$
-\frac{\pi^{2} a^{2} n^{2}}{l^{2}} T_{n}(-t)=\frac{a^{2}}{b^{2}} T_{n}^{\prime \prime}(t)+\frac{\pi^{2} a^{4} n^{2}}{b^{2} \ell^{2}} T_{n}(t)
$$


Thus, Eq. (3.34) is reduced to the fourth-order ordinary differential equation

$$
T_{n}^{(4)}(t)+\frac{2 \pi^{2} a^{2} n^{2}}{l^{2}} T_{n}^{\prime \prime}(t)+\frac{\pi^{4}\left(a^{4}-b^{4}\right) n^{4}}{l^{4}} T_{n}(t)=0
$$

with the initial conditions

$$
\begin{gathered}
T_{n}(0)=A_{n}, T_{n}^{\prime}(0)=B_{n}, T_{n}^{\prime \prime}(0)=-\frac{\pi^{2}\left(a^{2}+b^{2}\right) n^{2}}{l^{2}} A_{n}, \\
T_{n}^{(3)}(0)=-\frac{\pi^{2}\left(a^{2}-b^{2}\right) n^{2}}{l^{2}} B_{n} .
\end{gathered}
$$

It remains to consider various cases that may arise depending on the characteristic roots.

(1) For $b^{2}<a^{2}$,

$$
T_{n}(t)=A_{n} \cos \frac{\pi n \sqrt{a^{2}+b^{2}}}{l} t+\frac{l}{\pi n \sqrt{a^{2}-b^{2}}} B_{n} \sin \frac{\pi n \sqrt{a^{2}-b^{2}}}{l} t .
$$

(2) If $a^{2}=b^{2}$, then

$$
T_{n}(t)=A_{n} \cos \frac{\pi n a \sqrt{2}}{l} t+B_{n} t
$$

(3) Finally, the inequality $a^{2}<b^{2}$ leads to the result

$$
T_{n}(t)=A_{n} \cos \frac{\pi n \sqrt{a^{2}+b^{2}}}{l} t+\frac{l}{\pi n \sqrt{b^{2}-a^{2}}} B_{n} \sinh \frac{\pi n \sqrt{b^{2}-a^{2}}}{l} t \text {. }
$$

Of some interest is the equation

$$
u_{t}(t, x)=A u_{x x}\left(\frac{\alpha t+\beta}{\gamma t-\alpha}, x\right)
$$

with the hyperbolic involution

$$
f(t)=\frac{\alpha t+\beta}{\gamma t-\alpha}, \alpha^{2}+\beta \gamma \equiv \Delta^{2}>0, \quad \gamma \neq 0
$$

having two fixed points

$$
t_{0}=\frac{\alpha+\Delta}{\gamma}, \quad t_{1}=\frac{\alpha-\Delta}{\gamma} .
$$

The search of a solution in the region $(\alpha / \gamma, \infty) \times[0,1](\operatorname{or}(-\infty, \alpha / \gamma) \times[0,1])$ sat isfying the conditions

$$
u(t, 0)=u(t, l)=0, u\left(t_{0}, x\right)=\psi(x)\left(\text { or } u\left(t_{1}, x\right)=\psi(x)\right)
$$

leads to the relation

$$
T_{n}^{\prime}(t)=-\frac{A \pi^{2} n^{2}}{\ell^{2}} T_{n}\left(\frac{\alpha t+\beta}{\gamma t-\alpha}\right)
$$


which is a generalization of Eq. (2.1). Differentiation changes (3.37) to the form

$$
(\gamma t-\alpha)^{2} T_{n}^{\prime \prime}(t)+\frac{\Delta^{2} A^{2} \pi^{4} n^{4}}{\ell^{4}} T_{n}(t)=0 .
$$

The substitution $|\gamma t-\alpha|=$ exps permits integration of (3.38) in closed form. Omitting the calculations, we formulate a qualitative result.

THEOREM 3.11. The solution of problem $(3.35)-(3.36)$ is unbounded as $t \rightarrow \infty$. For

$$
\mathrm{n}>\frac{\ell}{\pi}\left(\frac{|\gamma|}{2|\mathrm{~A}| \Delta}\right)^{1 / 2}
$$

the functions $T_{n}(t)$ are oscillatory.

4. EQUATIONS WITH ROTATION OF THE ARGUMENT

An equation that contains, along with the unknown function $x(t)$ and its derivatives, the value $x(-t)$ and, possibly, the derivatives of $x$ at the point $-t$, is called a differential equation with reflection. An equation in which as well as the unknown function $x(t)$ and its derivatives, the values $x\left(\varepsilon_{1} t-\alpha_{1}\right), \ldots, x\left(\varepsilon_{m} t-\alpha_{m}\right)$ and the corresponding values of the derivatives appear, where $\varepsilon_{1}, \ldots, \varepsilon_{m}$ are $m$ th roots of unity and $\alpha_{1}, \ldots, \alpha_{m}$ are complex numbers, is called a differential equation with rotation. For $m=2$ this last definition includes the previous one. Linear first-order equations with constant coefficients and with reflection have been examined in detail in [5]. There is also an indication ( $p$. 169) that "the problem is much more difficult in the case of a differential equation with reflection of order greater than one". Meanwhile, general results for systems of any order with rotation appeared in [3], [4], [9], and [24].

Consider the scalar equation

$$
\begin{gathered}
\sum_{k=0}^{n} a_{k} x^{(k)}(t)=\sum_{k=0}^{n} b_{k} x^{(k)}(\varepsilon t)+\psi(t), \varepsilon^{m}=1 \\
x^{(k)}(0)=x_{k}, \quad k=0, \ldots, n-1
\end{gathered}
$$

with complex constants $a_{k}, b_{k}, \varepsilon$, then the method is extended to some systems with variable coefficients. Turning to $(4,1)$ and assuming that $\psi$ is smooth enough, we introduce the operators

$$
\begin{gathered}
A_{j}=\sum_{k=0}^{n} a_{k} \varepsilon^{-j k} \frac{d^{k}}{d t^{k}}, B_{j}=\sum_{k=0}^{n} b_{k} \varepsilon^{j k} \frac{d^{k}}{d t^{k}}, \\
(j=0, \ldots, m-1),
\end{gathered}
$$

and apply $A_{1}$ to the given equation 


$$
A_{0} x=\left(B_{0} x\right)(\varepsilon t)+\psi
$$

Since

$$
A_{1}\left[\left(B_{0} x\right)(\varepsilon t)\right]=\left(B_{1} B_{0} x\right)\left(\varepsilon^{2} t\right)+\left(B_{0} \psi\right)(\varepsilon t),
$$

we obtain

$$
A_{1} A_{0} x=\left(B_{1} B_{0} x\right)\left(\varepsilon^{2} t\right)+A_{1} \psi+\left(B_{0} \psi\right)(\varepsilon t),
$$

and act on this relation by $\mathrm{A}_{2}$. From

$$
\begin{aligned}
& A_{2}\left[\left(B_{1} B_{0} x\right)\left(\varepsilon^{2} t\right)\right]=\left(B_{2} B_{1} B_{0} x\right)\left(\varepsilon^{3} t\right)+\left(B_{1} B_{0} \psi\right)\left(\varepsilon^{2} t\right), \\
& A_{2}\left[\left(B_{0} \psi\right)(\varepsilon t)\right]=\left(A_{1} B_{0} \psi\right)(\varepsilon t)
\end{aligned}
$$

it follows that

$$
A_{2} A_{1} A_{0} x=\left(B_{2} B_{1} B_{0} x\right)\left(\varepsilon^{3} t\right)+A_{2} A_{1} \psi+\left(A_{1} B_{0} \psi\right)(\varepsilon t)+\left(B_{1} B_{0} \psi\right)\left(\varepsilon^{2} t\right) .
$$

Finally, this process leads to the ordinary differential equation

$$
\left(A_{0}^{(m-1)}-B_{0}^{(m-1)}\right) x=\sum_{j=0}^{m-1}\left(A_{1}^{(m-1-j)} B_{0}^{(j-1)} \psi\right)\left(\varepsilon^{j} t\right)
$$

where

$$
\begin{aligned}
& A_{i}^{(j)}=A_{j} A_{j-1} \cdots A_{i}, B_{i}^{(j)}=B_{j} B_{j-1} \cdots B_{i}, i \leq j \\
& A_{1}^{(0)}=B_{0}^{(-1)}=I,
\end{aligned}
$$

and $I$ is the identity operator. Thus, (4.1) is reduced to the ODE (4.2) of order mn. Io make the initial conditions for (4.2) agree with the original problem, it is necessary to attach to conditions (4.1) the additional relatiuns

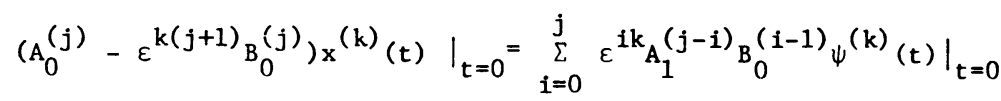

$$
\begin{aligned}
& (j=0, \ldots, m .2 ; k=0, \ldots, n-1) .
\end{aligned}
$$

System (4.3) has a unique solution for $x^{(k)}(0)(n \leq k \leq m n-1)$, iff

$$
a_{n}^{j} \neq\left(\varepsilon^{i_{b}}\right)_{n}^{j}, \quad(0 \leq i \leq m-1,1 \leq j \leq m-1) .
$$

These considerations enable us to formulate

THEOREM $4.1([9])$. If $\psi \varepsilon C^{(m-1) n}$ and inequalities $(4.4)$ are fulfilled, the solution of ordinary differential equation (4.2) with initial conditions (4.1) $-(4.3)$ satisfies problem (4.1).

THEOREM 4.2 ([9]). If $\varepsilon \neq 1$, the substitution

$$
y=x \exp (\alpha t / 1-\varepsilon)
$$

transforms the equation

$$
A y=\exp (\alpha t)(B y)(\varepsilon t)+\psi
$$


with operators $A$ and $B$ defined by $(4.1)$ to

$$
P x=(Q x)(\varepsilon t)+\psi \exp (-\alpha t / 1-\varepsilon),
$$

where $P$ and $Q$ are linear differential operators of order $n$ with constant coefficients $p_{k}, q_{k}$ and $p_{n}=a_{n}, q_{n}=h_{n}$.

COROLLARY. Under assumptions $(4.4)$ and $\varepsilon^{m}=1,(4.5)$ is reducible to a linear ordinary differential equation with constant coefficients.

RFMARK. Conditions (4.4) hold if, in particular, $\left|a_{n}\right| \neq\left|b_{n}\right|$. Theorems 4.1 ind 4.2 sharpen the corresponding results of [25] and [26] established for homogeneous equations $(4.1)$ and $(4.5)$ by operational methods under the restriction $\left|a_{n}\right|>\left|b_{n}\right|$.

EXAMPLE 4.1. The substitution $y=x$ expt reduces the equation [9]

$$
y^{\prime}(t)=\left(5 y(-t)+2 y^{\prime}(-t)\right) \exp 2 t, y(0)=y_{0}
$$

to the form

$$
x^{\prime}(t)+x(t)=7 x(-t)+2 x^{\prime}(-t), x(0)=y_{0} .
$$

Therefore (4.2) gives for $x(t)$ the ODE $x^{\prime \prime}-16 x=0$ with the initial conditions $x(0)=y_{0}, x^{\prime}(0)=-6 y_{0}$. The unknown solution is

$$
y(t)=y_{0}(5 \exp (-3 t)-\exp 5 t) / 4
$$

The analysis of the matrix equation

$$
\begin{gathered}
X^{\prime}(t)=A X(t)+\exp (\alpha t)\left[B X(\varepsilon t)+C X^{\prime}(\varepsilon t)\right], \\
X(0)=E
\end{gathered}
$$

with constant (complex) coefficients was carried out in [3]. The norm of a matrix is defined to be

$$
\| c||=\max _{i} \sum_{j}\left|c_{i j}\right|
$$

and $\mathrm{E}$ is the identity matrix.

THEOREM 4.3. ([3]). If $\varepsilon$ is a root of unity $(\varepsilon \neq 1),\|c\|<1$, and the matrix $A$ is commuting with $B$ and $C$, then problem $(4.6)$ is reducible to an ordinary linear system with constant coefficients.

The following particular case of Eq. (4.1) has been investigated in [27].

THEOREM 4.4. ([27]). Suppose we are given a differential equation with reflection of order $n$ with constant coefficients

$$
\sum_{k=0}^{n}\left[a_{k} x^{(k)}(t)+b_{k} x^{(k)}(-t)\right]=y(t) .
$$

We suppose that

$$
\text { (a) } a_{n}^{2}-b_{n}^{2} \neq 0,
$$


(b) $a_{j-k} a_{k}-b_{j-k} b_{k} \neq 0$ for $k=0,1, \ldots, n$ and $j=k+1, \ldots, k+n$,

(c) the polynomial $\sum_{j=0}^{n} \lambda_{2 j} t^{j}$ has simple roots $u_{q}$ only, where

$$
\lambda_{j}= \begin{cases}\sum_{k=0}^{j} c_{j k} \text { for } 0 \leq j \leq n, & \\ \sum_{j=j-n}^{n} c_{j k} \text { for } n \leq j \leq 2 n, & \end{cases}
$$

Then every solution of Eq. (4.8) is of the form

$$
\begin{gathered}
x(t)=(-1)^{n}\left(a_{n}^{2}-b_{n}^{2}\right)^{-1} \sum_{m=0}^{n}\left[(-1)^{m} a_{m} \tilde{x}(t)-b_{m} \tilde{x}(-t)\right]+ \\
\sum_{q=1}^{n} \sum_{k=0}^{n} c_{k} u_{q}^{k / 2}\left(a_{k} e^{\sqrt{u_{q}} t}-b_{k} e^{-\sqrt{u_{q}} t}\right),
\end{gathered}
$$

where the $C_{k}$ are arbitrary constants and $\tilde{x}(t)$ is a solution of the equation

$$
\prod_{q=1}^{n}\left(\frac{d^{2}}{d t^{2}}-u_{q}\right) \tilde{x}(t)=y(t) .
$$

THEOREM 4.5 ([9]). Suppose that the coefficienis of the equation

$$
\sum_{k=0}^{n} a_{k}(t) x^{(k)}(t)=x(\varepsilon t)+\psi(t), x^{(k)}(0)=x_{k}, k=0, \ldots, n-1(4.9)
$$

belong to $C^{(m-1) n}, \varepsilon^{m}=1, a_{n}(0) \neq 0$ and

$$
L_{j}=\sum_{k=0}^{n} \varepsilon^{-j k} a_{k}\left(\varepsilon^{j} t\right) d^{k} / d t^{k}, j=0, \ldots, m-1
$$

Then the solution of the linear ordinary differential equation

$$
\begin{gathered}
L_{0}^{(m-1)} x(t)=x(t)+\sum_{k=1}^{m-1}\left(L_{k}^{(m-1)} \psi\right)\left(\varepsilon^{k-1} t\right)+\psi\left(\varepsilon^{m-1} t\right), \\
\left(L_{k}^{(m-1)}=L_{m-1} L_{m-2} \cdots L_{k}, 0 \leq k \leq m-1\right)
\end{gathered}
$$

with the initial conditions

satisfies problem (4.9).

$$
\begin{aligned}
& x^{(k)}(0)=x_{k}(k=0, \ldots, n-1),\left.L_{0} x^{(k)}(t)\right|_{t=0}=\varepsilon^{k} x^{(k)}(0)+\psi^{(k)}(0), k=0, \ldots, \\
& n(m-1)-1
\end{aligned}
$$

PROOF. Applying the operator $L_{1}$ to $(4.9)$ and taking into account that 


$$
\left(L_{0} x\right)(\varepsilon t)=x\left(\varepsilon^{2} t\right)+\psi(\varepsilon t)
$$

we get

$$
L_{1} L_{0} x(t)=x\left(\varepsilon^{2} t\right)+L_{1} \psi(t)+\psi(\varepsilon t)
$$

and act on this equation by $L_{2}$ to obtain

$$
\mathrm{L}_{2} \mathrm{~L}_{1} \mathrm{~L}_{0} \mathrm{x}(\mathrm{t})=\mathrm{x}\left(\varepsilon^{3} \mathrm{t}\right)+\mathrm{L}_{2} \mathrm{~L}_{1} \psi(t)+\left(\mathrm{L}_{2} \psi\right)(\varepsilon t)+\psi\left(\varepsilon^{2} t\right)
$$

It is easy to verify the relations

$$
\left(L_{j} x\right)\left(\varepsilon^{j} t\right)=x\left(\varepsilon^{j+1} t\right)+\psi\left(\varepsilon^{j} t\right), j=0, \ldots, m-1 .
$$

In particular,

$$
\left(L_{m-1} x\right)\left(\varepsilon^{m-1} t\right)=x(t)+\psi\left(\varepsilon^{m-1} t\right) .
$$

Thus, the use of the operator $\mathrm{L}_{\mathrm{m}-1}$ at the conclusive stage yields (4.11).

THEOREM 4.6 ([9]). The system

$$
\operatorname{tAX}^{\prime}(t)+B X(t)=X(\varepsilon t)
$$

with constant matrices $A$ and $B$ is integrable in the closed form if $\varepsilon^{m}=1$, det $A \neq 0$. PROOF. For (4.12) the operators $L_{j}$ defined by formula (4.10) are

$$
\mathrm{L}_{j}=\mathrm{tAd} / \mathrm{dt}+\mathrm{B} \text {. }
$$

Hence, on the basis of the previous theorem, (4.12) is reducible to the ordinary system

$$
(\operatorname{tad} / d t+B)^{m} X(t)=X(t) .
$$

This is Euler's equation with matrix coefficients. Since its order is higher than that of (4.12) we substitute the general solution of (4.13) In (4.12) and equate the coefficients of the like terms in the corresponding logarithmic sums to find the additional unknown constants.

EXAMPLE 4.2. We connect with the equation [9]

$$
t x^{\prime}(t)-2 x(t)=x(\varepsilon t), \varepsilon^{3}=1
$$

the relation

$$
(t d / d t-2)^{3} x(t)=x(t) .
$$

The substitution of its general solution

$$
x(t)=C_{1} t^{3}+t^{3 / 2}\left(C_{2} \sin \left(\frac{\sqrt{3}}{2} \ln t\right)+C_{3} \cos \left(\frac{\sqrt{3}}{2} \ln t\right)\right)
$$

into (4.14) gives $C_{2}=C_{3}=0$. A solution of $(4.14)$ is

$$
\mathrm{x}=\mathrm{Ct} \mathrm{t}^{3} \text {. }
$$

THEOREM 4.7 ([9]). The system

$$
\operatorname{tAX}^{\prime}(t)=B X(t)+t X(\varepsilon t)
$$


with constant coefficients $A$ and $B$, det $A \neq 0$ and $\varepsilon^{m}=1$ is integrable in closed form and has a solution

$$
X(t)=P(t) t^{A^{-1} B}
$$

where the matrix $P(t)$ is a finite linear combination of exponential functions.

PROOF. The transition from $(4.15)$ to an ordinary equation is realized by means of the operators

$$
L_{j}=\varepsilon^{-j}\left(A d / d t-t^{-1} B\right), j=0, \ldots, m-1
$$

in consequence of which we obtain the relation

$$
\left(A d / d t-t^{-1} B\right)^{m} X(t)=\varepsilon^{m(m-1) / 2} X(t) .
$$

Since $\varepsilon^{m(m-1) / 2}= \pm 1$, it takes the form

$$
\prod_{k=1}^{m}\left[\operatorname{Ad} / d t-\left(\varepsilon_{k} E+t^{-1} B\right)\right] x(t)=0
$$

where $\varepsilon_{k}$ are the m-order roots of 1 or -1 . The solutions of the equations

$$
\mathrm{AX}^{\prime}(t)=\left(\varepsilon_{k} E+t^{-1} B\right) X(t)
$$

are matrices

$$
x_{k}(t)=\exp \left(\varepsilon_{k} t^{-1}\right) t^{A^{-1} B}, k=1, \ldots, m .
$$

Their linear combination represents the general solution of (4.15).

EXAMPLE 4.3. In accordance with (4.17), to the equation [9]

$$
t x^{\prime}(t)=3 x(t)+t x(-t)
$$

there correspond two ordinary relations

$$
x^{\prime}(t)=\left(3 t^{-1}+1\right) x(t), x^{\prime}(t)=\left(3 t^{-1}-1\right) x(t)
$$

We substitute into $(4.18)$ the linear combination of their solutions

$$
x(t)=t^{3}\left(c_{1} \exp (i t)+C_{2} \exp (-i t)\right)
$$

and find $C_{2}=i C_{1}$. A solution of $(4.18)$ is

$$
x(t)=C t^{3}(\sin t+\cos t) \text {. }
$$

Biological models of ten lead to systems of delay or functional differential equations (FDE) and to questions concerning the stability of equilibrium solutions of such equations. The monographs [28] and [29] discuss a number of examples of such models which describe phenomena from population dynamics, ecology, and physiology. The work [29] is mainly devoted to the analysis of models leading to reducible FDE. A necessary and sufficient condition for the reducibility of a FDE to a system of ordinary differential equations is given by the author of [30]. His method is fre- 
quently used to study FDE arising in biological models. We omit these topics and refer to a recent paper [31]. For the study of analytic solutions to FDE, which will be the main topic in the next part of our paper, we also mention survey [32].

\section{REFERFNCES}

1. WIENER, J. Differential equations with involutions, Differencial'nye Uravnen1ja $\underline{6}$ (1969), 1131-1137.

2. WIENER, J. Differential equations in partial derivatives with involutions, Differencial'nye Uravnenija ? (1970), 1320-1322.

3. WIENER, J. Differential equations with periodic transformations of the argument, Izv. Vyš̌. Učebn. Zaved. Radiofizika 3 (1973), 481-484.

4. WIENER, J. Investigation of some functional differential equations with a regular singular point, Differencial nye Uravnenija 10 (1974), 1891-1894.

5. PRZEWORSKA-ROLEWICZ, D. Equations with transformed argument. An algebraic approach, Panstwowe Wydawnictwo Naukowe, Warszawa, 1973.

6. SARKOVSKII, A.N. Functional-differential equations with a finite group of argument transformations, Asymptotic behavior of solutions of functionaldifferential equations, Akad. Nauk Ukrain. SSR, Inst.Mat., Kiev (1978), $118-142$.

7. KURDANOV, Kh.Yu. The influence of an argument deviation on the behavior of solutions of differential equations, Differencial'nye Uravnenija 15 (1979), 944.

8. KISIELEWICZ, M. (Editor) Functional differential systems and related topics, Proceedings of the First International Conference held at Blaźejewko, May 19-26, 1979. Higher College of Engineering, Jnstitute of Mathematics and Physics, Zielona Góra (Poland), 1980.

9. COOKE, K. and WIENER, J. Distributional and analytic solutions of functional differential equations, J. Math. Anal. Appl. 98 (1984), 111-129.

10. WIENER, J. and AFTABIZADEH, A.R. Boundary value problems for differential equations with reflection of the argument, Internat. J. Math. \& Math.Sci. (to appear).

11. SilbersteIN, L. Solution of the equation $f^{\prime}(x)=f(1 / x)$, Philos. Magazine 30 (1940), 185-186.

12. WIENER, J. On Silberstein's functional equation, Učen. Zap. Ryazan. Pedagog. Inst. $41(1966), 5-8$.

13. LUČIĆ, R. On a functional differential equation, Publ. Electrotechn. Fac. Univ. Belgrade, Ser. Math. Phys. 338-352 (1971), 55-56.

14. SHISHA, O. and MEHR, C.B. On involutions, J. Nat. Bur. Stand. 71B (1967), 19-20.

15. BOGDANOV, Yu.S. On the functional equation $x^{n}=t$, DAN BSSR $\underline{5}(1961), 235-237$.

16. LUČIC, R. On a class of functional differential equations, Publ. Electrotechn. Fac. Univ. Belgrade, Ser. Math. Phys. 461-497 (1974), 31-32.

17. LUČIĆ, R. Functional differential equations whose arguments form a finite group, Publ. Electrotechn. Fac. Univ. Belgrade, Ser. Math. Phys. 498-541 (1975), 133-135.

18. CODDINGTON, E.A. and LEVINSON, N. Theory of ordinary differential equations, McGraw-Hill, New York, 1955. 
19. KULLER, R.G. On the differential equation $\mathrm{f}^{\prime}=\mathrm{f} \circ \mathrm{g}$, where $\mathrm{g} \circ \mathrm{g}=\mathrm{I}$, Math. Mag. 42 (1969), 195-200.

20. CASTELAN, W.G. and INFANTE, E.F. On a functional equation arising in the stability theory of difference-differential equations, Quart. Appl. Math. 35 (1977), 311-319.

21. BELLMAN, R. Introduction to matrix analysis, McGraw-Hi11, New York, 1960.

22. REPIN, I.M. Quadratic Liapunov functionals for systems with delays, Prikl. Matem. Mekh. 29 (1965), 564-566.

23. DATKO, R. An algorithm for computing Liapunov functionals for some differential difference equations, in Ordinary differential equations, 1971 NRL-MRC Conference, Academic Press (1972), 387-398.

24. WIENER, J. Periodic mappings in the study of functional differential equations, Differencial'nye Uravnenija 3, Ryazan (1974), 34-45.

25. BRUWIER, L. Sur l'application du calcul cymbolique á la iresolution d'equations fonctionnelles, Bu11. Soc. R. Sci. Liege 17 (1948), 220-245.

26. VALEEV, K.G. On solutions of some functional equations, Issled. po Integro-diff. Uravn. V Kirgizii 5 $(1968), 85-89$.

27. MAZ̈BIC-KULMA, B. On an equation with reflection of order $\mathrm{n}$, Studia Math. $\underline{35}$ (1970), 69-76.

28. CUSHING, J.M. Integrodifferential equations and delay models in population dynamics, in "Lecture Notes in Biomathematics, No. 20", Springer-Verlag, Ber1in, $197 \overline{7}$.

29. MCDONALD, N. Time lags in biological models, in "Lecture Notes in Biomathematics, No. 27", Springer-Verlag, Berlin, 1978.

30. FARGUE, D.M. Réducibilité des systémes héréditaires a des systémes dynamiques, C. R. Acad. Sci. Paris Ser. B 277 (1973), 471-473.

31. BUSENBERG, S. and TRAVIS, C. On the use of reducible-functional differential equations in biological models, J. Math. Anal. App1. 89 (1982), 46-66.

32. SHAH, S.M. and WIENER, J. Distributional and entire solutions of ordinary differential and functional differential equations, Internat. J. Math. \& Math. Sci. 6(2), (1983), 243-270. 


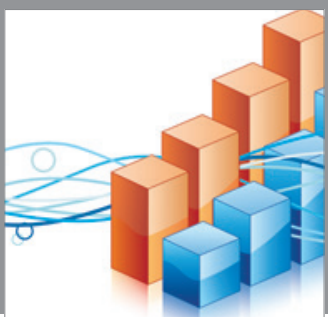

Advances in

Operations Research

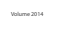

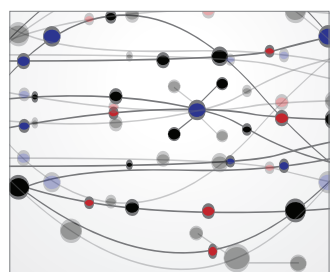

\section{The Scientific} World Journal
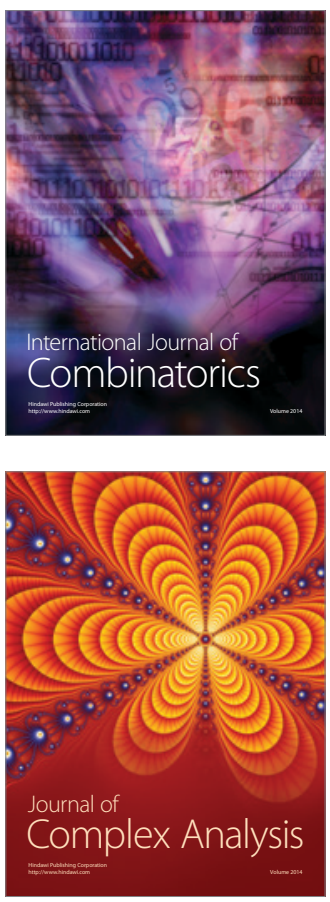

International Journal of

Mathematics and

Mathematical

Sciences
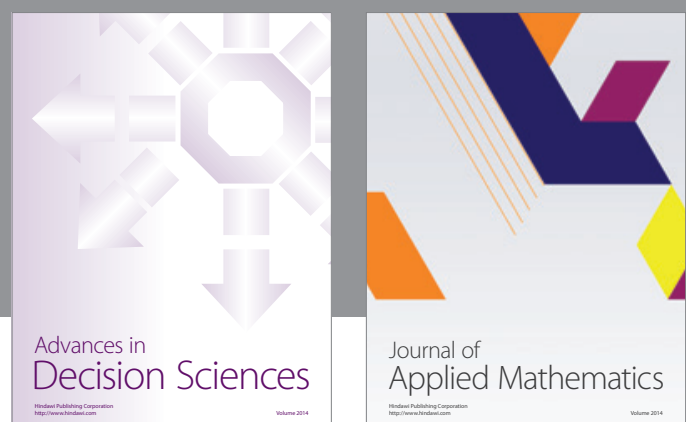

Journal of

Applied Mathematics
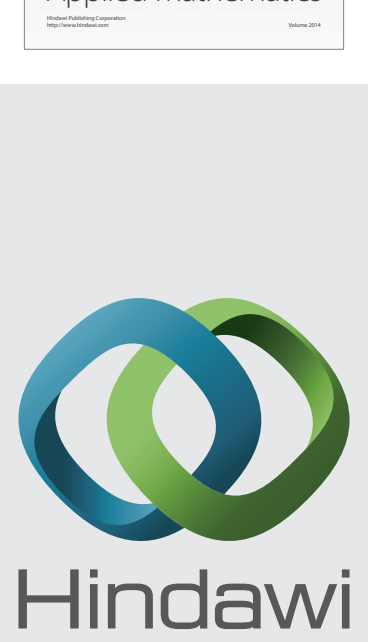

Submit your manuscripts at http://www.hindawi.com
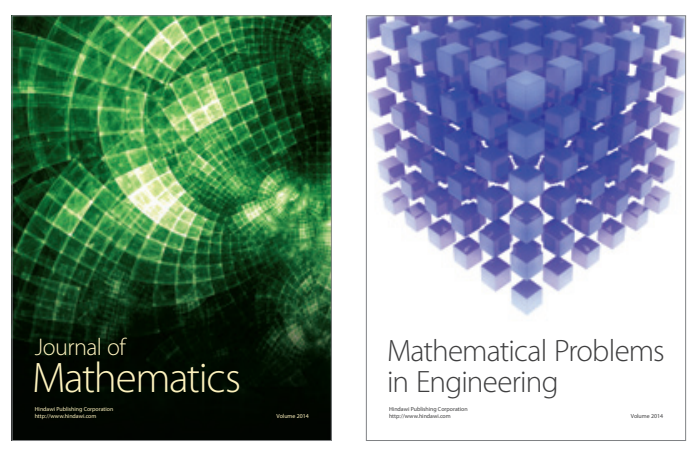

Mathematical Problems in Engineering
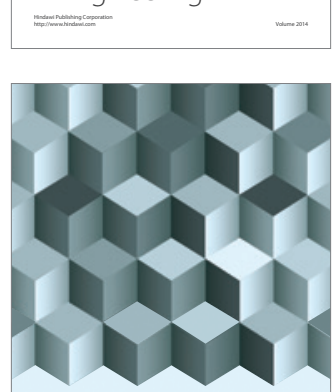

Journal of

Function Spaces
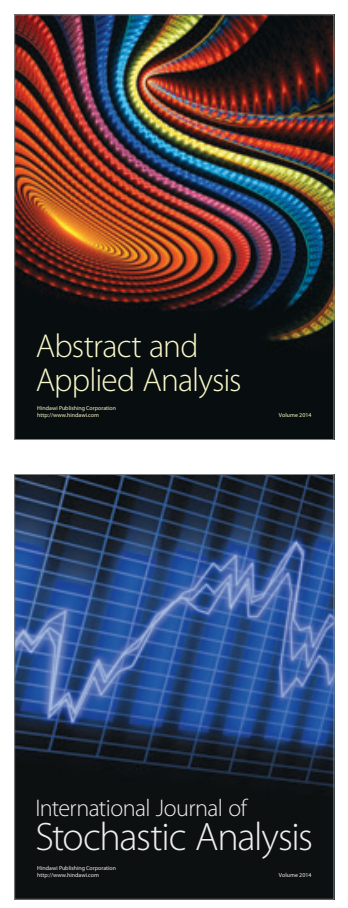

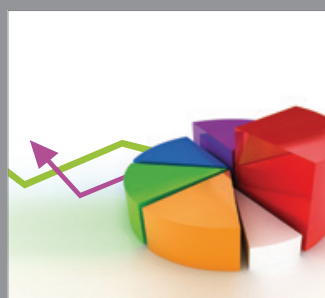

ournal of

Probability and Statistics

Promensencen
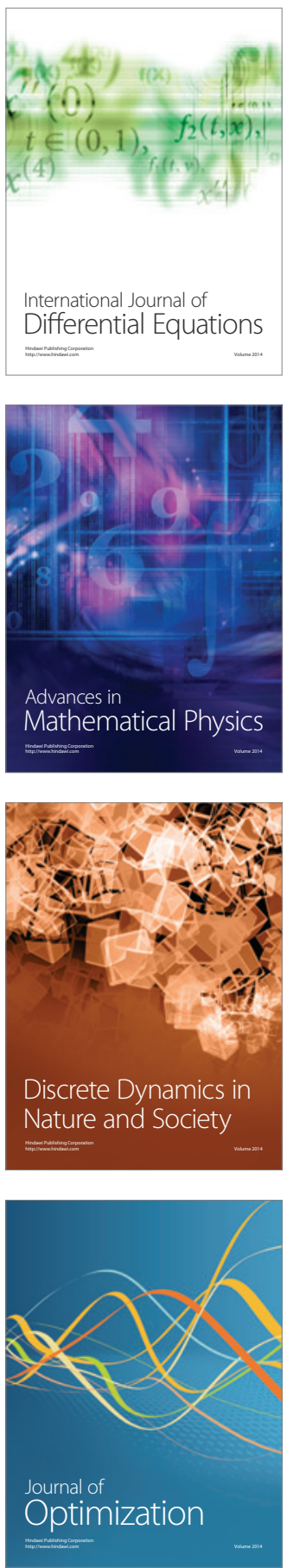